\title{
FROM REAL TO COMPLEX SIGN PATTERN MATRICES
}

\author{
Carolyn A. Eschenbach, Frank J. Hall and Zhongshan Li
}

This paper extends some fundamental concepts of qualitative matrix analysis from sign pattern classes of real matrices to sign pattern classes of complex matrices. A complex sign pattern and its corresponding sign pattern class are defined in such a way that they generalize the definitions of a (real) sign pattern and its corresponding sign pattern class. A survey of several qualitative results on complex sign patterns is presented. In particular, sign nonsingular complex patterns are investigated. The type of region in the complex plane representing the distribution of the determinants of the matrices in the sign pattern class of a sign nonsingular complex pattern is identified. Cyclically nonnegative complex patterns and complex patterns that are signature similar to nonnegative patterns are characterized. Extensions of sign stable and sign semistable patterns from the real to the complex case are given. Results on ray patterns are also obtained. Finally, many open questions are mentioned.

\section{INTRODUCTION}

Interest in qualitative matrix analysis was stimulated, in part, by the need to analyse certain dynamical systems for which only qualitative information was available. Such systems arise in economics, ecology, biology, chemistry, mechanics and energy planning when only the directions of certain effects are known. Much interest has recently been focused on qualitative matrix methods, since certain combinatorial results can be obtained from them.

To date, qualitative matrix analysis involves the study of properties that a real matrix may or may not have based strictly upon knowledge of the signs of the entries of the matrix. A matrix whose entries are from the set $\{+,-, 0\}$ is called a (real) sign pattern matrix. Associated with each $n$-by- $n$ sign pattern matrix $A=\left(a_{i j}\right)$ is a natural class of real matrices, called the sign pattern class of $A$, defined by

$$
Q(A)=\left\{B \in M_{n}(R) \mid \operatorname{sgn} b_{i j}=a_{i j} \text { for all } i \text { and } j\right\}
$$

If $P$ is a property referring to a real matrix, then the sign pattern matrix $A$ is said to require $P$ if every matrix in the sign pattern class of $A$ satisfies $P$, and $A$ is said to allow

Received 20th May, 1997

Copyright Clearance Centre, Inc. Serial-fee code: 0004-9729/98 \$A2.00+0.00. 
$P$ if some matrix in the sign pattern class satisfies $P$. In this paper, we do not attempt to give an exhaustive set of references for real sign pattern matrices. However, we point out that the recent book by Brualdi and Shader [3] includes extensive references.

Complex matrices arise naturally in theory as well as in many applications. For example, linear dynamical systems with complex entries occur in quantum mechanics [9]. Hence, it would be useful to investigate properties of complex matrices based upon the signs of the real and imaginary parts of their entries. One goal of this paper is to survey some fundamental properties of qualitative matrix analysis that can be extended from sign pattern classes of real matrices to sign pattern classes of complex matrices. For $n$-by- $n$ sign pattern matrices $A_{1}$ and $A_{2}$, we define a complex sign pattern matrix to be $A=A_{1}+i A_{2}$, and the sign pattern class of $A$ is defined by

$$
Q(A)=\left\{B_{1}+i B_{2} \mid B_{1} \in Q\left(A_{1}\right) \text { and } B_{2} \in Q\left(A_{2}\right)\right\} \text {. }
$$

For brevity, we often use complex (or real) pattern or complex (or real) sign pattern to mean a complex (or real) sign pattern matrix.

Given two angles $\alpha \leqslant \beta$, we define a sector in the complex plane to be the set of all nonzero complex numbers whose arguments are in the interval from $\alpha$ to $\beta$, where the interval could be closed, and if $\alpha<\beta$, the interval could be closed, open, or half-open. We denote the corresponding sector by $\left[e^{i \alpha}, e^{i \beta}\right],\left(e^{i \alpha}, e^{i \beta}\right)$ or, $\left[e^{i \alpha}, e^{i \beta}\right)$ or $\left(e^{i \alpha}, e^{i \beta}\right]$, respectively. Whenever $\alpha=\beta$, the sector becomes a ray, denoted by $e^{i \alpha}$. Note that the ray $e^{i \alpha}$ is the set of all nonzero complex numbers whose arguments are equal to $\alpha$, while the sector $\left[e^{i 0}, e^{i 2 \pi}\right]$ is the same as $\mathbb{C} \backslash\{0\}$. For any integer $n$, we associate the rays $e^{2 n \pi i}, e^{(2 n+1) \pi i}, e^{(4 n+1)(\pi / 2) i}$ and $e^{(4 n+3)(\pi / 2) i}$ with,,$+- i$ and $-i$, respectively. Clearly, each nonzero entry in a complex sign pattern is a sector that represents either a quadrant or one of the rays,,$+- i$ or $-i$. As usual, by a quadrant in the complex plane, we mean an open quadrant that does not include the axes.

The definitions relating to the cycles and paths in an $n$-by- $n$ complex pattern $A=\left(a_{i j}\right)$ are analogous to the corresponding definitions in a real sign pattern. In particular, we define a path of length $k$ from $i_{1}$ to $i_{k+1}$ to be a formal product of the form $\gamma=a_{i_{1} i_{2}} a_{i_{2} i_{3}} \cdots a_{i_{k} i_{k+1}}$. Similarly, we define a simple cycle of length $k$ ( $k$-cycle) to be a formal product of the form $\gamma=a_{i_{1} i_{2}} a_{i_{2} i_{3}} \ldots a_{i_{k} i_{1}}$, where the indices $i_{1}, i_{2}, \ldots, i_{k}$ are distinct. By ap $(\gamma)$ we mean the actual product of the entries in $\gamma$, where the multiplication is carried out in the usual way. For example, if $\gamma=\left[e^{i \alpha_{1}}, e^{i \beta_{1}}\right]\left[e^{i \alpha_{2}}, e^{i \beta_{2}}\right]$, then $\operatorname{ap}(\gamma)=\left[e^{i\left(\alpha_{1}+\alpha_{2}\right)}, e^{i\left(\beta_{1}+\beta_{2}\right)}\right]$. If $\gamma$ is a cycle and ap $(\gamma)$ equals,,$+- i$ or $-i$, we say $\gamma$ is a positive, negative, positive pure imaginary or negative pure imaginary cycle, respectively. In the remainder of this paper, when we say cycle we mean a nonzero cycle, that is, a cycle that contains no zero entries.

Some known results concerning real sign pattern classes easily extend to the complex sign pattern classes. For example, sign singularity of an $n$-by- $n$ sign pattern matrix 
(real or complex), meaning that every matrix in the sign pattern class is singular, is equivalent to the existence of a $p$-by- $q$ submatrix consisting entirely of zeros, where $p+q \geqslant n+1$ This result is true for matrices over an arbitrary field, and follows from a theorem of Frobenius-König (see [2, p.6], and [6]). However, some characterisations do not carry over to the complex sign pattern classes. It is well known that a real sign pattern matrix $A$ is sign nonsingular, that is, every matrix in the sign pattern class of $A$ is nonsingular, if and only if there is at least one nonzero term in the determinant of $A$, denoted by $\operatorname{det} A$, and all nonzero terms in $\operatorname{det} A$ have the same sign [1]. To illustrate that this characterisation does not generalise to sign nonsingular complex patterns, consider the following example. Let

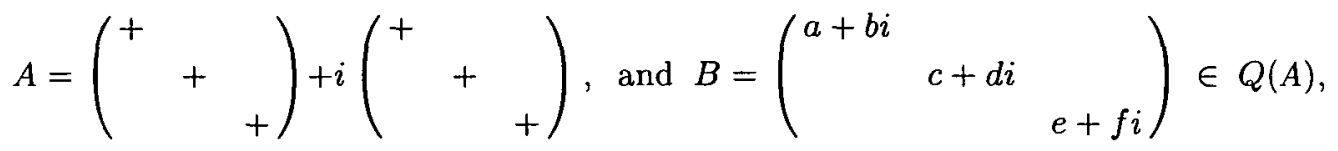

where $a, b, c, d, e$ and $f$ are positive real numbers. Clearly, $A$ is sign nonsingular, however,

$$
\operatorname{det} B=(a c e-a d f+\cdots)+i(b c e-b d f+\cdots),
$$

which has mixed signs in each of the real and imaginary parts. Two fundamental questions arise:

(i) How are the sign nonsingular complex sign patterns characterised? and

(ii) How can the region in the complex plane consisting of the determinantal values of the complex matrices $B$ in the sign pattern class of a sign nonsingular complex pattern $A$ be located?

The latter question is addressed in section 2 of this paper, while the former question remains an open research problem.

In section 3 we investigate cyclically nonnegative complex sign patterns, and also characterise the complex patterns that are signature similar to nonnegative patterns. In section 4 , we extend sign stability to the class of complex patterns whose diagonal entries have negative real parts, and we extend a characterisation from real sign semistable patterns to complex patterns. In section 5 , we obtain results on ray patterns. Finally, in section 6 , we mention several general open questions.

\section{Sign NONSINGULARITY}

Let $A$ be a real $n$-by- $n$ sign nonsingular pattern. Then $\operatorname{det} A=+\operatorname{or} \operatorname{det} A=-$. If $B \in Q(A)$, then $\operatorname{det} B \in(0, \infty)$ whenever $\operatorname{det} A=+$, and $\operatorname{det} B \in(-\infty, 0)$ whenever $\operatorname{det} A=-$. The goal of this section is to establish general determinantal regions for sign nonsingular complex patterns. 
If $A$ is an $n$-by- $n$ complex pattern, and if $B \in Q(A)$ with $\operatorname{det} B=b$, then for any positive number $k, \operatorname{det}(\sqrt[n]{k} B)=k b$. If $A$ is sign nonsingular, since $(\sqrt[n]{k} B) \epsilon$ $Q(A)$, it follows that every nonzero number lying on the ray originating at the origin and passing through $b$ can be achieved as the determinant of some matrix in $Q(A)$. We call such a ray a determinantal ray of $A$. The set $S_{A}=\{\operatorname{det} B \mid B \in Q(A)\}$ is called the determinantal region of the complex sign pattern $A$.

THEOREM 2.1. If $A$ is a square sign nonsingular complex sign pattern, then the determinantal region of $A$ is a sector in the complex plane.

Proof: Let $B, B_{1} \in Q(A)$, and define $C_{t} \in Q(A)$ by $C_{t}=t B+(1-t) B_{1}$ for $0 \leqslant t \leqslant 1$. Since the determinant is a function that depends continuously upon the entries of a matrix, the curve $C$ represented by the set of points $\left\{\operatorname{det} C_{t} \mid 0 \leqslant t \leqslant 1\right\}$ between $\operatorname{det} B$ and $\operatorname{det} B_{1}$ is continuous. Since $A$ is sign nonsingular, $C$ does not pass through the origin. Let $\theta$ and $\theta_{1}$ be the arguments of the points $\operatorname{det} B$ and $\operatorname{det} B_{1}$. We may assume that $\theta \leqslant \theta_{1}<\theta+2 \pi$. By the continuity of the curve $C$, we see that either the sector from $\theta$ to $\theta_{1}$ or the sector from $\theta_{1}$ to $\theta+2 \pi$ is contained in the determinantal region of $A$. Since $B$ and $B_{1}$ are arbitrary matrices in $Q(A)$, it follows that the determinantal region of $A$ is a sector.

We now illustrate Theorem 2.1 with several examples. We let $I_{n}$ be the diagonal sign pattern of order $n$ with + entries on the main diagonal.

EXAMPLE 2.2.

(i) If $A=I_{2}+i\left(\begin{array}{ll}+ & 0 \\ 0 & 0\end{array}\right)$, then $S_{A}$ is the first quadrant.

(ii) If $A=I_{2}+i I_{2}$, then $S_{A}$ is the first two quadrants, together with the positive imaginary axis. In other words $S_{A}$ is the open sector from 0 to $\pi$.

(iii) If $A=I_{3}+i I_{3}$, then $S_{A}$ is the open sector from 0 to $(3 \pi / 2)$.

(iv) If $A=I_{4}+i I_{4}$, then $S_{A}$ is the open sector from 0 to $2 \pi$.

(v) If $A=I_{5}+i I_{5}$, then $S_{A}$ is the whole plane excluding the origin.

(vi) If $A=\left(\begin{array}{cc}i & i \\ - & +\end{array}\right)=\left(\begin{array}{cc}0 & 0 \\ - & +\end{array}\right)+i\left(\begin{array}{cc}+ & + \\ 0 & 0\end{array}\right)$, then $S_{A}$ is the positive imaginary axis.

(vii) If $A=\left(\begin{array}{ll}+ & - \\ + & +\end{array}\right)+i\left(\begin{array}{cc}+ & 0 \\ 0 & -\end{array}\right)$, then $S_{A}$ is the open sector from $-\pi / 2$ to $\pi / 2$.

Two important related research questions are:

(i) If $A$ is sign nonsingular, are the boundaries of $S_{A}$ always axes in the complex plane? and 
(ii) What is an effective characterisation of sign nonsingular complex patterns?

It is well known that if $A$ is a 3-by-3 sign nonsingular real pattern, then $A$ contains at least one zero entry. In our next example, we illustrate that this is not the case for complex sign patterns.

EXAMPLE 2.3. Let

$$
A=\left(\begin{array}{ccc}
+ & + & + \\
- & + & 0 \\
- & - & +
\end{array}\right)+i\left(\begin{array}{lll}
0 & 0 & 0 \\
0 & 0 & + \\
0 & 0 & 0
\end{array}\right)=A_{1}+i A_{2} .
$$

Then the real part of $\operatorname{det} A$ is signed since $\operatorname{Re}(\operatorname{det} A)=+$, and it follows that $\operatorname{det} B \neq 0$ for any $B \in Q(A)$. Thus $A$ is an entrywise nonzero 3-by-3 sign nonsingular pattern.

In general, if $A$ is an $n$-by- $n$ sign nonsingular real pattern, then $A$ contains at least $\left(\begin{array}{c}n-1 \\ 2\end{array}\right)$-entries [15]. A natural question is: If $A$ is a sign nonsingular complex pattern of order $n \geqslant 4$, is there a minimum number of 0 entries, and, if so, what is the minimum number?

It is of interest to determine the possible determinantal regions for arbitrary complex patterns. For example, if

$$
A=\left(\begin{array}{ccc}
+ & + & 0 \\
+ & + & 0 \\
0 & 0 & (+)+i
\end{array}\right)
$$

then $A$ is not sign nonsingular, and the determinantal region of $A$ is the union of the first and third quadrants, together with the origin. Note that this region is a union of sectors plus the origin.

\section{CyClic Nonnegativity}

We begin this section by describing positive and negative cycles in a complex pattern. To this end, we note that if $\gamma$ contains at least one complex entry that represents a quadrant, then $\gamma$ is not a positive or a negative cycle. Thus, if $\gamma$ is a positive or a negative cycle, then $\gamma$ consists of a product of rays from the set $\{+,-, i,-i\}$.

The conjugate of a sector is defined in the usual way, for example, the conjugate of $\left[e^{i \alpha}, e^{i \beta}\right)$ is $\left(e^{-i \beta}, e^{-i \alpha}\right]$. Hence if $A=A_{1}+i A_{2}$, then $A^{*}$ is defined in the natural way as the conjugate transpose of $A$, so that $A^{*}=A_{1}^{T}-i A_{2}^{T}$. A complex signature pattern is a diagonal pattern $S$ whose diagonal entries come from the set $\{+,-, i,-i\}$. If $S$ is a signature pattern, then $S^{-1}=S^{*}$ is the signature pattern such that

$$
S^{-1} S=S S^{-1}=\left(\begin{array}{ccc}
+ & & \bigcirc \\
& \ddots & \\
\bigcirc & & +
\end{array}\right) \text {. }
$$


If $A$ is an $n$-by- $n$ complex pattern, and $S$ is a signature pattern of order $n$, then $S^{-1} A S$ is called a signature similarity of $A$. It is easy to show that any signature similarity of $A$ preserves the qualitative cycle structure of $A$. That is, if $\gamma$ is a cycle in $A$, and if $\gamma_{s}$ is the corresponding cycle in $S^{-1} A S$, then $\operatorname{ap}(\gamma)=\operatorname{ap}\left(\gamma_{s}\right)$.

As in the case of real sign patterns, a cyclically nonnegative complex pattern is a square pattern whose cycles are positive. Similarly, a cyclically positive complex pattern is an entrywise nonzero cyclically nonnegative pattern.

THEOREM 3.1. If $A$ is an $n$-by- $n$ cyclically positive complex sign pattern, then $A$ is signature similar to the all + pattern of order $n$.

ProOF: We use induction on the order $k$ of the matrix. Clearly, the result is true for $k=1$. Now assume the result holds for $k=n-1$, and let $A$ be an $n$-by- $n$ cyclically positive complex pattern. By the induction hypothesis, there exists a signature pattern $S_{1}$ such that

$$
S_{1}^{-1} A S_{1}=\left(\begin{array}{cc}
A_{1} & A_{2} \\
A_{3} & A_{4}
\end{array}\right),
$$

where $A_{1}$ is the all + pattern of order $n-1$, and where $A_{4}=\left(a_{n n}\right)=(+)$ is a 1-by-1 positive diagonal entry of $A$ ( $A$ is cyclically positive).

From the discussion preceding this theorem, we know that $A$ cyclically positive implies that $S_{1}^{-1} A S_{1}$ is cyclically positive. Thus $\left(S_{1}^{-1} A S_{1}\right)_{n i}\left(S_{1}^{-1} A S_{1}\right)_{i j}\left(S_{1}^{-1} A S_{1}\right)_{j n}=$ + for all indices $1 \leqslant i, j \leqslant n$. Since $\left(S_{1}^{-1} A S_{1}\right)_{i j}$ is an entry in $A_{1}$, we see that $\left(S_{1}^{-1} A S_{1}\right)_{i j}=+$, and it follows that $\left(S_{1}^{-1} A S_{1}\right)_{n i}\left(S_{1}^{-1} A S_{1}\right)_{j n}=+$ for all $1 \leqslant i, j<n$. Consequently, $\left(S_{1}^{-1} A S_{1}\right)_{n i}=x$ for all $i=1, \ldots, n-1$ and $\left(S_{1}^{-1} A S_{1}\right)_{j n}=y$ for all $j=1, \ldots, n-1$, for some $x$ and $y$ in the set $\{+,-, i,-i\}$, where $x y=+$. Finally, if

$$
S_{2}=\left(\begin{array}{cccc}
+ & & & \\
& \ddots & & \\
& & + & \\
& & & x
\end{array}\right)
$$

then it is clear that $S_{2}^{-1}\left(S_{1}^{-1} A S_{1}\right) S_{2}$ is the all + pattern of order $n$.

Corollary 3.2. An irreducible complex sign pattern $A$ is cyclically nonnegative if and only if $A$ is signature similar to a nonnegative pattern.

Proof: Let $A$ be an $n$-by- $n$ irreducible cyclically nonnegative complex pattern. Then, since $A$ is irreducible, every nonzero entry of $A$ lies on some simple cycle. Further, since $A=\left(a_{i j}\right)$ is cyclically nonnegative, we know that no entry in $A$ represents a quadrant, that is, each $a_{i j} \in\{+,-, 0, i,-i\}$.

Now if $P_{1}=a_{i k_{1}} \ldots a_{k_{p} j}$ and $P_{2}=a_{i m_{1}} \ldots a_{m_{q} j}$ are two paths in $A$ from $i$ to $j$, then $A$ irreducible implies that there is at least one path from $j$ to $i$, say, 
$P=a_{j n_{1}} \ldots a_{n_{s} i}$. Since $P_{1} P$ and $P_{2} P$ are products of simple cycles in $A$, it follows that ap $\left(P_{1} P\right)=\operatorname{ap}\left(P_{2} P\right)=+$. Thus ap $\left(P_{1}\right)=$ ap $\left(P_{2}\right)$, and we conclude that all paths in $A$ from $i$ to $j$ for any pair of indices $i$ and $j$ have the same sign.

Define the matrix $A^{\prime}=\left(a_{i j}^{\prime}\right)$ by

$$
a_{i j}^{\prime}= \begin{cases}a_{i j} & \text { if } a_{i j} \neq 0 \\ \operatorname{ap}\left(P_{i j}\right) & \text { if } a_{i j}=0, \text { where } P_{i j} \text { is a path from } i \text { to } j .\end{cases}
$$

Then $A^{\prime}$ is entrywise nonzero and cyclically positive. From Theorem 3.1 , there exists a signature pattern $S$ such that $S^{-1} A^{\prime} S$ is the all + pattern, and, hence, $S^{-1} A S$ is nonnegative.

Since the converse of corollary 3.2 is clear, the proof is now complete.

A natural question now arises, namely: What can we say about reducible cyclically nonnegative complex patterns? Recall that every reducible pattern $A$ is permutation similar to a pattern of the form

$$
\left(\begin{array}{cccc}
A_{11} & A_{12} & \ldots & A_{1 m} \\
& A_{22} & \ldots & A_{2 m} \\
& & \ddots & \vdots \\
& & & A_{m m}
\end{array}\right),
$$

where the diagonal blocks are irreducible. This is called the Frobenius normal form of $A$, and the $A_{i i}$ 's are called the irreducible components of $A$. Since every cycle of a reducible matrix occurs in some irreducible component, a complex pattern is cyclically nonnegative if and only if each irreducible component is cyclically nonnegative.

It is also of interest to characterise the $n$-by- $n$ complex patterns that are signature similar to nonnegative patterns. If $A$ is signature similar to a nonnegative pattern, then $B \in Q(A)$ is signature similar to a nonnegative matrix. From the Perron-Frobenius theory, we know that the spectral radius $\rho(B)$ is an eigenvalue of $B$. Thus every $B \in Q(A)$ has its spectral radius as an eigenvalue, and we say that $A$ requires the Perron property. Real sign patterns that require/allow the Perron property are discussed in [4]. Later, in Theorem 3.5, we show that cyclically nonnegative complex patterns are precisely those that require the Perron property.

If a complex sign pattern $A$ is cyclically nonnegative, then for each $k$ and $j$, $a_{k j} a_{j k}=+$ or 0 , that is, $\overline{a_{j k}}=a_{k j}$ or one of $a_{k j}$ and $a_{j k}$ is 0 . Hence, when we form the sum $A+A^{*}$, by adding corresponding entries, we obtain a complex sign pattern. Further, we note that if $A$ is cyclically nonnegative, then $A$ is a subpattern of $A+A^{*}$, meaning that $A$ can be obtained by setting some $k(k \geqslant 0)$ entries in $A+A^{*}$ to 0 . 
THEOREM 3.3. Let $A$ be an $n-b y-n$ complex sign pattern. Then the following are equivalent:

(i) $A$ is signature similar to a nonnegative pattern;

(ii) $A$ is cyclically nonnegative and $A+A^{*}$ is signature similar to a nonnegative pattern;

(iii) $A$ and $A+A^{*}$ are both cyclically nonnegative.

ProOF: (i) $\Rightarrow$ (ii). If $S^{-1} A S$ is nonnegative for some signature pattern $S$, then $S^{-1} A^{*} S$ is nonnegative. Hence, $S^{-1}\left(A+A^{*}\right) S$ is nonnegative.

(ii) $\Rightarrow$ (iii) is clear.

(iii) $\Rightarrow$ (i). Suppose $A$ and $A+A^{*}$ are cyclically nonnegative. Since $A+A^{*}=$ $\left(A+A^{*}\right)^{*}$, the Frobenius normal form of $A+A^{*}$ is given by

$$
P^{*}\left(A+A^{*}\right) P=\left(\begin{array}{cccc}
A_{11} & & \bigcirc & \\
& A_{22} & & \\
\bigcirc & & \ddots & \\
& & & A_{m m}
\end{array}\right)
$$

for some permutation pattern $P$. Further, since each irreducible component $A_{i i}$ is cyclically nonnegative, by corollary 3.2 , there exists a signature matrix $S_{i i}$ such that $S_{i i}^{-1} A_{i i} S_{i i}$ is nonnegative. Therefore, if

$$
S=\left(\begin{array}{ccc}
S_{11} & & \bigcirc \\
& \ddots & \\
\bigcirc & & S_{m m}
\end{array}\right)
$$

then $S^{-1} P^{*}\left(A+A^{*}\right) P S$ is nonnegative. Consequently, $P S^{-1} P^{*}\left(A+A^{*}\right) P S P^{*}$ is nonnegative. However, $P S P^{*}$ is a signature matrix, and it follows that $A+A^{*}$ is signature similar to a nonnegative pattern. Finally, since $A$ is a subpattern of $A+A^{*}$, it is clear that $A$ is signature similar to a nonnegative matrix.

EXAMPLE 3.4. Let

$$
A=\left(\begin{array}{ccccc}
+ & 0 & - & i & + \\
i & + & 0 & - & 0 \\
- & i & + & 0 & - \\
0 & 0 & 0 & + & -i \\
0 & 0 & 0 & i & 0
\end{array}\right)
$$

Since $A$ and $A+A^{*}$ are cyclically nonnegative, it follows that $A$ is signature similar to a nonnegative pattern.

When we say that we are emphasising a simple cycle $\gamma$ in a complex pattern $A$, we mean that we are choosing a matrix $B$ in $Q(A)$ such that the entries along $\gamma$ each have 
modulus one, and all other nonzero entries in $B$ have modulus $\varepsilon$, for some arbitrarily small $\varepsilon>0$. To prove the necessary condition stated in Theorem 3.5, we shall need the type of perturbation arguments used in [4, pp.303-304]. These arguments use the fact that the eigenvalues depend continuously upon the entries of a matrix.

Theorem 3.5. An $n$-by- $n$ complex sign pattern A requires the Perron property if and only if $A$ is cyclically nonnegative.

Proof: Suppose a complex pattern $A$ has a simple $k$-cycle $\gamma=a_{i_{1} i_{2}} a_{i_{2} i_{3}} \cdots a_{i_{k} i_{1}}$ such that ap $(\gamma) \neq+$. By emphasising the cycle $\gamma$, we can find a matrix $B \in Q(A)$ such that $c=b_{i_{1} i_{2}} b_{i_{2} i_{3}} \ldots b_{i_{k} i_{1}} \neq 1$ (but $|c|=1$ ). Then $k$ of the eigenvalues of $B$ are arbitrarily close to the complex $k$-th roots of $c$ and the other $n-k$ eigenvalues of $B$ are arbitrarily close to zero. Since $c$ is not positive, $B$ does not have the Perron property.

Now suppose $A$ is cyclically nonnegative. Then each irreducible component of $A$ is signature similar to a nonnegative sign pattern matrix (by corollary 3.2 ), and, thus, $A$ requires the Perron property.

\section{STABILITY}

A matrix $B$ is said to be stable if each eigenvalue of $B$ has a negative real part. Complex (real) sign patterns $A$ that require stability are called sign stable, and complex (real) patterns that allow stability are called potentially stable. Considerable research has been done to characterise real sign stable patterns. In [13] and [14], the class of matrices that are sign stable is identified for the case in which a real sign pattern $A$ has a negative diagonal. To date, there is no effective characterisation of real potentially stable patterns, and perhaps complex qualitative analysis may shed some light on this problem. Since a complex pattern $A$ is sign stable if and only if each irreducible component of $A$ is sign stable, we turn our attention to characterising irreducible sign stable complex patterns.

To prove the following lemma, we can modify the argument used in the proof of Theorem 3.5. Here we choose a matrix $B$ in $Q(A)$ by emphasising a simple 2-cycle $\gamma$ so that the product of the entries in $B$ along $\gamma$ is not equal to -1 but has modulus 1 .

Lemma 4.1. If a complex sign pattern $A$ is sign stable, then $A$ has no simple 2-cycle of the form $\gamma=(s+t i)(u+v i)$, where $s, t \neq 0$ or $u, v \neq 0$.

Lemma 4.2 gives the necessary conditions for complex sign stability. The necessary conditions for real sign stability are given in Lemma 5.1 of [13]. Condition (i) of [13, Lemma 5.1] has been modified here to allow some complex diagonal entry that has a negative real part. All other conditions in Lemma 4.2 are the same as the conditions for real sign stability, and can be proved using similar arguments as in [13], together with Lemma 4.1. 
Lemma 4.2. If $A=A_{1}+i A_{2}$ is an $n$-by- $n$ complex sign stable pattern, then

(i) all diagonal entries in $A_{1}$ are nonpositive, with at least one diagonal entry negative;

(ii) all simple 2-cycles in $A$ are negative;

(iii) there are no simple cycles in $A$ of length greater than 2; and

(iv) $A$ is sign nonsingular.

Recall that $A$ is combinatorially symmetric if $a_{i j} \neq 0$ implies $a_{j i} \neq 0$. Since $[\mathbf{1 3}$, Lemma 5.2] involves only the location of the zero and nonzero entries of a matrix, the result is also true for complex sign patterns. We state this result as Lemma 4.3, and omit the proof, which can be found in [11].

LEMmA 4.3. If $A$ is irreducible and has no simple cycles of length greater than two, then $A$ is combinatorially symmetric with $n-1$ nonzero terms above (below) the main diagonal.

Clearly, negative 2-cycles have one of the following forms: $(i)(i),(-i)(-i),(-)(+)$, or $(+)(-)$. Consequently, we can extend the characterisation of real sign stable patterns with negative diagonal entries, found in [13] and [14], to complex patterns.

THEOREM 4.4. Let $A=A_{1}+i A_{2}$ be an $n$-by- $n$ irreducible complex sign pattern matrix, where all the diagonal entries of $A_{1}$ are negative. Then $A$ is sign stable if and only if

(i) all simple 2-cycles in $A$ are negative; and

(ii) there are no simple cycles in $A$ of length greater than 2.

ProOF: Necessity follows from Lemma 4.2.

For sufficiency, let $B \in Q(A)$. We construct a diagonal matrix $D=\left(d_{i j}\right)$ as follows: Set $d_{i j}=0$ for all indices $i \neq j$, and let $d_{11}=1$. For all indices $i, j=1, \ldots, n$, choose $d_{i i}=\alpha d_{j j}$, where $\alpha=-\overline{b_{j i}} / b_{i j}$, whenever $b_{i j}$ is nonzero.

Then conditions (i) and (ii) together with Lemma 4.3 guarantee that $d_{i i}>0$ for all $i=1, \ldots, n$. Since $A$ is a tree sign pattern matrix ( $A$ is irreducible, combinatorially symmetric and has no cycles of length greater than two), initially specifying $d_{11}$ uniquely determines $D$.

Now, when $i \neq j$ and $b_{i j} \neq 0$, the $(i, j)$ entry of $D B+B^{*} D$ is

$$
d_{i i} b_{i j}+\overline{b_{j i}} d_{j j}=\frac{-\overline{b_{j i}}}{b_{i j}} d_{j j} b_{i j}+\overline{b_{j i}} d_{j j}=\left(-\overline{b_{j i}}+\overline{b_{j i}}\right) d_{j j}=0
$$

Also, the $(i, i)$ entry of $D B+B^{*} D$ is $d_{i i} b_{i i}+\bar{b}_{i i} d_{i i}=\left(b_{i i}+\bar{b}_{i i}\right) d_{i i}$, which is negative since the diagonal entries of $B$ have negative real part. Thus, $D B+B^{*} D$ is a diagonal matrix with negative diagonal entries. By Lyapunov's Theorem, $B$ is a stable matrix. 
A general characterisation of real sign stable patterns is given in [10]. The characterisation in Theorem 4.4 extends the class of real sign stable patterns with negative diagonal entries identified in [13] and [14]. A natural generalisation of this is to find a characterisation of arbitrary complex sign stable patterns.

We note that if $A=A_{1}+i A_{2}$ is an $n$-by- $n$ sign pattern matrix with the property that all the diagonal entries of $A_{1}$ are negative, then clearly, $A$ is potentially stable. This follows because we can choose $B \in Q(A)$ so that the entries on the diagonal of $B$ are large in modulus in comparison with the remaining entries in $B$. Consequently, a natural question is: What are the necessary and sufficient conditions for $A$ to be potentially stable?

We say that an $n$-by- $n$ complex (real) sign pattern matrix $A$ is sign semistable if, for each $B \in Q(A), \operatorname{Re}(\lambda) \leqslant 0$ for all $\lambda$ in the spectrum of $B$. The necessary and sufficient conditions for real semistable sign patterns are given in [14]. Using these conditions, we obtain the following characterisation of irreducible sign semistable complex patterns.

Corollary 4.5. Let $A=A_{1}+i A_{2}$ be an $n$-by- $n$ irreducible, complex sign pattern. Then $A$ is sign semistable if and only if

(i) all diagonal entries in $A_{1}$ are nonpositive;

(ii) all simple 2-cycles in $A$ are negative; and

(iii) there are no simple cycles in $A$ of length greater than 2.

Proof: The necessity of the conditions is clear.

For sufficiency, let $B \in Q(A)$. Set $\widetilde{B}=B-\varepsilon I$, where $\varepsilon$ is some arbitrarily small positive number, and $I$ is the identity matrix of order $n$. Then $\tilde{B} \in Q(\tilde{A})$, and $\tilde{A}$ satisfies the conditions stated in Theorem 4.4. Thus $\widetilde{B}$ is stable, and for sufficiently small $\varepsilon>0$, the eigenvalues of $B$ are close to the eigenvalues of $\widetilde{B}$. Consequently, $B$ is semistable (see [14, Lemma 5].

\section{RAY PATTERNS}

A ray pattern was defined and ray nonsingularity was investigated in [12]. By a ray pattern we mean a matrix each of whose entries is either 0 or a ray $e^{i \theta}$. Associated with each $n$-by- $n$ ray pattern $A=\left(a_{i j}\right)$ is the qualitative class of $A$, defined by

$$
\begin{aligned}
Q(A)=\left\{B \in M_{n}(\mathbb{C}) \mid b_{i j}=0 \text { if and only if } a_{i j}=0, \arg b_{i j}=\arg a_{i j}\right. \\
\text { for all } \left.i \text { and } j \text { where } a_{i j} \neq 0\right\} .
\end{aligned}
$$

For ray patterns, the definitions of the terms require (allow) a property $\mathrm{P}$, simple cycle, and cyclically nonnegative are the same as for complex sign patterns. However, we note 
that positive 2-cycles in a ray pattern have the general form $\left(e^{i \theta}\right)\left(e^{-i \theta}\right)$, and negative 2-cycles have the general form $\left(e^{i \theta}\right)\left(e^{i(\pi-\theta)}\right)$.

For a ray pattern $A$, we define $A^{*}$ in the usual way as $(\bar{A})^{T}$. A signature ray pattern $S$ is a diagonal ray pattern with nonzero diagonal entries.

The arguments and results of sections 3 and 4 can easily be modified to apply to ray patterns. For example, corollary 3.2 for ray patterns becomes:

Theorem 5.1. An irreducible ray pattern $A$ is cyclically nonnegative if and only if $A$ is signature similar to a nonnegative pattern.

The irreducible cyclically nonnegative complex patterns form a proper subclass of the class of irreducible cyclically nonnegative ray patterns. However, the respective reducible classes are clearly different, since the strictly upper triangular blocks in the Frobenius normal form allow different types of entries.

When we say a ray pattern $A$ is ray stable, we mean that every matrix $B \in Q(A)$ is stable. For ray patterns, Theorem 4.4 on stability can be rephrased as follows:

TheOREM 5.2. Let $A$ be an $n$-by- $n$ irreducible ray pattern, where all the diagonal entries of $A$ have arguments in the left half plane. Then $A$ is ray stable if and only if

(i) all simple 2-cycles in $A$ are negative; and

(ii) there are no simple cycles in $A$ of length greater than 2.

To prove the sufficiency of the conditions in this latter theorem, we choose $\alpha=$ $\left|b_{j i} / b_{i j}\right|$ and proceed as in the proof of Theorem 4.4.

Although we have greater variability with the negative 2-cycles in ray patterns than in complex sign patterns, the diagonal entries in the latter could represent the second or the third quadrants, so that the irreducible sign stable complex patterns do not form a proper subclass of the class of irreducible ray stable patterns.

Complex patterns and ray patterns are two generalisations of real sign patterns. More generally, one can consider a sector pattern where each entry is either 0 or an arbitrary sector in the complex plane.

\section{Concluding Remarks}

In the first five sections of this paper, we mentioned several open questions related to the topics covered in these sections. We now give some additional open questions. For example, it is of interest to characterise the $n$-by- $n$ complex sign patterns that require/allow $k$ real eigenvalues, where $k$ is a positive integer less than or equal to $n$. Since the nonreal eigenvalues of real matrices occur in complex conjugate pairs, it is clear that if $A$ is an $n$-by- $n$ real sign pattern matrix, and if $n$ is odd, then $A$ requires 
a real eigenvalue. We note that the real patterns of even order that require a real eigenvalue are characterised in [5]. Since complex sign patterns of odd order may or may not require a real eigenvalue, any characterisation of complex patterns that require one real eigenvalue must include odd as well as even orders. Another spectral question is: What are the complex sign patterns that require repeated eigenvalues?

Given a complex pattern $A$ that allows nonsingularity, what are the possible complex patterns of the inverses of the invertible matrices $B$ in $Q(A)$ ? A natural related question is: When does $A$ require/allow $B^{-1} \in Q(A)$ ? We note that real sign patterns that require $B^{-1} \in Q(A)$ are called self-inverse sign patterns and are characterised in [7], and real sign patterns that allow this property are discussed in [8]. Another question is: What complex sign patterns allow an invertible matrix $B \in Q(A)$ such that the inverse of $B$ is in $Q\left(A^{*}\right)$ ? In particular, what complex sign patterns arise as the sign patterns of unitary matrices?

\section{REFERENCES}

[1] L. Bassett, J. Maybee and J. Quirk, 'Qualitative economics and the scope of the correspondence principle', Econometrica 36 (1968), 544-563.

[2] R. Brualdi and H. Ryser, Combinatorial matrix theory (Cambridge University Press, Cambridge, 1991).

[3] R. Brualdi and B. Shader, Matrices of sign-solvable linear systems (Cambridge University Press, Cambridge, 1995).

[4] C. Eschenbach and C. Johnson, 'A combinatorial converse to the Perron frobenius theorem', Linear Algebra Appl. 136 (1990), 173-180.

[5] C. Eschenbach and C. Johnson, 'Sign patterns that require real, nonreal or pure imaginary eigenvalues', Linear and Multilinear Algebra 29 (1991), 299-311.

[6] C. Eschenbach and C. Johnson, 'Sign patterns that require repeated eigenvalues', Linear Algebra Appl. 190 (1993), 169-179.

[7] C. Eschenbach, F. Hall and C. Johnson, 'Self-Inverse Sign Patterns', IMA Vol. Math. Appl. 50 (1993), 245-256.

[8] C. Eschenbach, F. Hall and Z. Li, 'Some sign patterns that allow a real inverse pair $B$ and $B^{-1}$, Linear Algebra Appl. 252 (1997), 299-321.

[9] R. Feynman, R. Leighton and M. Sands, The Feynman lectures on physics, Vol. III, Quantum Mechanics (Addison-Wesley, Reading, Mass., 1965).

[10] C. Jeffries, V. Klee and P. van den Driessche, 'When is a matrix sign stable?', Canad. J. Math. 29 (1977), 315-326.

[11] J. Maybee, 'New generalizations of Jacobi matrices', SIAM J. Appl. Math. 14 (1966), 1032-1037.

[12] J. McDonald, D. Olesky M. Tsatsomeros and P. van den Driessche, 'Ray patterns of matrices and nonsingularity', Linear Algebra Appl. 267 (1997), 359-373. 
[13] J. Maybee and J. Quirk, 'Qualitative problems in matrix theory', SIAM Review 11 (1969), 30-51.

[14] J. Quirk and R. Rupport, 'Qualitative economics and the stability of equilibrium', Rev. Econom. Stud. 32 (1965), 311-326.

[15] C. Thomassen, 'Sign-nonsingular matrices and even cycles in directed graphs', Linear Algebra Appl. 75 (1986), 27-41.

Department of Mathematics and Computer Science

Georgia State University

Atlanta GA 30303

United States of America 